\title{
Convective Effects on MHD Flow and Heat Transfer between Vertical Plates Moving in Opposite Direction and Partially Filled with a Porous Medium
}

\author{
V. G. Gupta1, Ajay Jain², Abhay Kumar Jha ${ }^{3}$ \\ ${ }^{1}$ Department of Mathematics, University of Rajasthan, Jaipur, India \\ ${ }^{2}$ Department of Mathematics, Regional College for Education, Research and Technology, Jaipur, India \\ ${ }^{3}$ Department of Mathematics, JECRC University, Jaipur, India \\ Email: ajay.jains2009@gmail.com
}

Received 13 January 2016; accepted 22 February 2016; published 25 February 2016

Copyright (C) 2016 by authors and Scientific Research Publishing Inc.

This work is licensed under the Creative Commons Attribution International License (CC BY). http://creativecommons.org/licenses/by/4.0/

(c) $\underset{\mathrm{EY}}{\mathrm{B}}$ Open Access

\begin{abstract}
The present paper, a theoretical analysis of steady fully developed flow and heat transfer of two immiscible magneto hydrodynamic and viscous fluid, partially filled with porous matrix and partially with clear fluid bounded by two vertical plates, has been discussed, when both the plates are moving in opposite directions. The plates are maintained at unequal temperatures. The Brinkman-extended Darcy model has described the momentum transfer in a porous medium. The effect of various parameters and Darcy number are discussed in the flow field and the temperature profiles numerically and are expressed by graphs. The non-dimensional governing momentum and energy equations are analytically solved by applying the homotopy perturbation technique and the method of ordinary differential equation. It is observed that magnetic parameter (M) has a retarding effect on the main flow velocity and is to enhance the temperature distribution, whereas the reversal phenomenon occurs for the Darcy dissipation parameter (Da). The skin-friction component has also been determined and is presented with the help of a table. The magnetic parameter $(\mathrm{M})$ reduces the skin friction coefficient for clear fluid region and is to increase the skin friction coefficient for porous region. It is also evident from table that getting bigger the width of the clear fluid layer increases the skin friction. The skin friction coefficient on both the plates (comparing at $\mathrm{y}=\mathbf{0}$ and at $\mathrm{y}=1$ for $\mathrm{A}$ or $\mathrm{B}$ ) increases when those are heated.
\end{abstract}

\section{Keywords}

Free Convection, MHD Flow, Porosity, Skin Friction 


\section{Introduction}

The convective flow and heat transfer of electrically conducting fluid in the channel have been an important research topic for the last few decades under the influence of magnetic field because of its applications in magneto-hydrodynamics (MHD) power generators, solar technology, the dispersion of metals, application in fusion reactors, aerodynamic heating, petroleum industry, crude oil purification, fluid droplet sprays and many more. Many relevant, pertinent studies have been reported as Hartmann [1] who discussed the effect of magnetic field on the flow field in the channel when both of the walls are electrically insulated. Rossow [2] obtained exact solution of Navier-Stokes equations and Maxwell's equation for the special case when the ratio of magnetic Reynold number and the viscosity is one. Such types of above research problems are extended with an external magnetic field and comprehensive reviewed by Helliwell [3], Attia and Kotb [4], Malathy and Srinivas [5], Singh et al. [6]. All these above pertain to one-fluid flow problems.

Heat transfer aspects associated with flow systems comprising multi layer flow in a region, part of which is occupied by porous matrix and part by clear fluid under the effect of transverse magnetic field, have a pivotal importance due to its wide range of applications in both geophysical and industrial environments, including such as thermal energy storage system, flow and heat transfer behavior of lubricants in a porous journal bearings and porous rollers, oil recovery, groundwater hydrology, petroleum reservoir engineering and many others in which a porous matrix is set up adjacent to clear fluid.

The comprehensive view of technological point in above fluid mechanics, some slip of the fluid over the fluid-porous boundary may occur so slip velocity ought to be found in empirical way. A series of work has been investigated on the problem of immiscible fluid. Beavers and Joseph [7] reported that the velocity gradient on the fluid side of the interface is proportional to the slip velocity at the interface. Taylor [8] and Richardson [9] extended the investigation of fluid flow by Darcy number. Degen et al. [10] studied the two-layer system with time dependent pattern. Lohrasbi and Sahai [11] analyzed the effect of MHD heat transfer in parallel plates when one phase of layers is conducted. Further, Vafai and Kim [12] dealt with porous region using the so-called Brinkman-Forchheimer-extended Darcy equation. Vafai and Kim [13], Kuznetsov [14], Alazmi and Vafai [15], Valencia-Lopez and Ochoa Tapia [16], Malashetty and Leela [17] and Umavathi et al. [18]-[20] reportd the substantial work on interfacial fluid problems with porous layers using different parameters and boundary conditions.

Furthermore, the study of fully developed forced convection in porous matrix is applied and in progressed by Vafai and Tien [21], Kaviany [22] who expressed theoretical and analytic solution of Brinkman-Darcy model. Comprehensive study in this area is made by Nield and Bejan [23] and presented the convection and heat flux in porous media with the effects of solid boundaries and inertial forces on flow and heat transfer in porous medium. Alkam et al. [24] investigated the convection and heat flux in channels with two porous layers attached to the walls. Rudraiah \& Nagraj [25], Kaviany [26] and Backermann et al. [27] discussed analytically the free convection flow of interfacial fluid of two parallel plates filled a pot with a Darcy porous layer. Singh et al. [28] presented an analytical solution of Brinkman-Forchheimer equations-extended Darcy model, which shows the effect of Brinkman (shear), and Forchheimer (inertia) in the porous drenched fluid. Khalili et al. [29] governed the flow by Darcy-Forchheimer equation and hence applied into superposed fluid and porous layers with vertical through-flow. Umavathi et al. [30] have presented analytical and theoretical solutions for unsteady two composite fluid flows and heat flux transfer in a horizontal channel. Stamenkovic [31] carried out the pioneer work in the study of MHD flow of immiscible, electrically conducting fluids between isothermal and insulated moving plates in the presence of an inclined magnetic field. Gupta et al. [32] described the viscous and Darcy dissipation effect on flow field through vertical plates partially filled with porous matrix when both the plates are moving in opposite directions. Simon and Shagaiya [33] observed the convective flow of two immiscible fluid and heat transfer with porous media along an inclined channel with pressure gradient. Mateen [34] considered the effect of magnetic field and ratio of viscosity on the flow and the temperature of two immiscible fluids through horizontal channel. He [35], [36] and Biazar and Ghazvini [37] perceived the solution of non-linear coupled equations by homotopy perturbation technique.

The proposed study of MHD multi fluid flow and heat transfer are through two vertical plates partially filled with porous matrix when both the plates are moving in opposite directions.

\section{Mathematical Analysis}

Consider a channel of an incompressible, viscous, steady and electrically conducting MHD multi fluid flow past 
between two vertical plates partially filled with porous media and partially with a clear fluid having an interface is discussed, when both the plates are moving in opposite directions and one plate is heated and the other is cooled. The $\bar{x}$ axis is taken along one of the plate and $\bar{y}$ axis normal to it and the applied magnetic field $\beta o$ along the $\bar{y}$ axis. The electrical field owing to the polarization of charges and Hall effect is taken negligible. However, the effect of Julian heat dissipation is incorporated. The velocity of the plate in the clear fluid region and the velocity of the plate in the porous region is $\bar{u}_{f}$ and $\bar{u}_{p}$ respectively.

$\bar{T}_{f}=\bar{T}_{c}+A\left(\bar{T}_{h}-\bar{T}_{c}\right)$ and $\bar{T}_{p}=\bar{T}_{c}+B\left(\bar{T}_{h}-\bar{T}_{c}\right)$ are the temperature of the plates situated at $\bar{y}=0$ and $\bar{y}=L$ respectively.

Under Bousinesque approximation, the flow of clear fluid and porous medium is governed by the following equations:

Clear Fluid Region:

(a) Momentum equation

$$
\frac{\mathrm{d}^{2} \bar{u}_{f}}{\mathrm{~d} \bar{y}^{2}}+\frac{g \beta}{v}\left(\bar{T}_{f}-\bar{T}_{c}\right)-\frac{\sigma \beta o^{2} \bar{u}_{f}}{v}=0
$$

(b) Energy equation

$$
\left(\frac{\mathrm{d} \bar{u}_{f}}{\mathrm{~d} \bar{y}}\right)^{2}+\frac{K}{v} \frac{\mathrm{d}^{2}}{\mathrm{dy} \bar{y}^{2}}\left(\bar{T}_{f}-\bar{T}_{c}\right)+\frac{\sigma \beta o^{2} \bar{u}_{f}^{2}}{v}=0
$$

Pore Fluid Region:

(a) Momentum equation

$$
\frac{\mathrm{d}^{2} \bar{u}_{p}}{\mathrm{~d} \bar{y}^{2}}-\frac{\bar{u}_{p}}{\bar{K}_{p}}+\frac{g \beta}{v}\left(\bar{T}_{p}-\bar{T}_{c}\right)-\frac{\sigma \beta o^{2} \bar{u}_{p}}{v}=0
$$

(b) Energy equation

$$
\left(\frac{\mathrm{d} \bar{u}_{p}}{\mathrm{~d} \bar{y}}\right)^{2}+\frac{\bar{u}_{p}^{2}}{\bar{K}_{p}}+\frac{K}{v} \frac{\mathrm{d}^{2}}{\mathrm{~d} \bar{y}^{2}}\left(\bar{T}_{p}-\bar{T}_{c}\right)+\frac{\sigma \beta o^{2} \bar{u}_{p}^{2}}{v}=0
$$

The corresponding boundary conditions are [boundary at interface $\bar{y}=\bar{d}$ is defined by Kim and Russel [38]]:

$$
\begin{gathered}
\bar{u}_{f}=\frac{g \beta L^{2}\left(\bar{T}_{h}-\bar{T}_{c}\right) \bigcup_{0}}{v}, \bar{T}_{f}=\bar{T}_{c}+A\left(\bar{T}_{h}-\bar{T}_{c}\right) \text { at } \bar{y}=0, \\
\bar{u}_{p}=\frac{g \beta L^{2}\left(\bar{T}_{h}-\bar{T}_{c}\right) \bigcup_{0}}{v}, \bar{T}_{p}=\bar{T}_{c}+B\left(\bar{T}_{h}-\bar{T}_{c}\right) \text { at } \bar{y}=L \\
\bar{y}=\bar{d}, \bar{u}_{f}=\bar{u}_{p}, \frac{\mathrm{d} \bar{u}_{f}}{\mathrm{~d} \bar{y}}=\frac{d \bar{u}_{p}}{\mathrm{~d} \bar{y}}, \bar{T}_{f}=\bar{T}_{p}, \frac{\mathrm{d} \bar{T}_{f}}{\mathrm{~d} \bar{y}}=\frac{\mathrm{d} \bar{T}_{p}}{\mathrm{~d} \bar{y}}
\end{gathered}
$$

Introducing dimensions by using the following transformation:

$$
\begin{aligned}
& D a=\frac{\bar{K}_{p}}{L^{2}}, y=\frac{\bar{y}}{L}, u_{f}=\frac{v \bar{u}_{f}}{g \beta L^{2}\left(\bar{T}_{h}-\bar{T}_{c}\right)}, d=\frac{\bar{d}}{L} \\
& u_{p}=\frac{v \bar{u}_{p}}{g \beta L^{2}\left(\bar{T}_{h}-\bar{T}_{c}\right)}, \theta_{f}=\frac{\bar{T}_{f}-\bar{T}_{c}}{\bar{T}_{h}-\bar{T}_{c}}, \theta_{p}=\frac{\bar{T}_{p}-\bar{T}_{c}}{\bar{T}_{h}-\bar{T}_{c}}
\end{aligned}
$$

And

$$
\beta_{\mathrm{u}}=\frac{g^{2} \beta^{2} L^{4}\left(\bar{T}_{h}-\bar{T}_{c}\right)}{K v}, M^{2}=\frac{\sigma \beta o^{2} L^{2}}{v}
$$

So Equation (1) to Equation (4) become: 


$$
\begin{gathered}
\frac{\mathrm{d}^{2} u_{f}}{\mathrm{~d} y^{2}}-M^{2} u_{f}+\theta_{f}=0 \\
\frac{\mathrm{d}^{2} \theta_{f}}{\mathrm{~d} y^{2}}+\beta_{u}\left(\frac{\mathrm{d} u_{f}}{\mathrm{~d} y}\right)^{2}+\beta_{u} M^{2} u_{f}^{2}=0 \\
\frac{\mathrm{d}^{2} u_{p}}{\mathrm{~d} y^{2}}-M_{1}^{2} u_{p}+\theta_{p}=0 \\
\frac{\mathrm{d}^{2} \theta_{p}}{\mathrm{~d} y^{2}}+\beta_{u}\left(\frac{\mathrm{d} u_{p}}{\mathrm{~d} y}\right)^{2}+\beta_{u} M_{1}^{2} u_{p}^{2}=0
\end{gathered}
$$

where $M_{1}^{2}=\frac{1}{D a}+M^{2}$

The Brinkman-extended Darcy law (Darcy [39] governs the momentum transfer of permeable domain).

The boundary conditions on velocity are no-slip conditions requiring that the velocity must be same as that at the plate. In addition, to maintain the continuity of velocity, shear stress, temperature and heat flux at the interface is assumed so boundary conditions (5) in non-dimension form are:

$$
\begin{aligned}
& y=0: u_{f}=U_{0}, \theta_{f}=A \\
& y=1: u_{p}=-U_{0}, \theta_{p}=B \\
& y=d: u_{f}=u_{p}, \frac{\mathrm{d} u_{f}}{\mathrm{~d} y}=\frac{\mathrm{d} u_{p}}{\mathrm{~d} y} \\
& y=d: \theta_{f}=\theta_{p}, \frac{\mathrm{d} \theta_{f}}{\mathrm{~d} y}=\frac{\mathrm{d} \theta_{p}}{\mathrm{~d} y}
\end{aligned}
$$

where $\bar{K}_{p}$ the permeability of porosity, Da the Darcy parameter, $K$ the thermal conductivity, $M$ the magnetic parameter (Hartmann number), $\beta_{u}$ the Buoyancy parameter, $\beta$ the coefficient of thermal expansion, $\mu$ is the viscosity, $\beta_{0}$ is the magnetic field intensity, $\sigma$ is the electric conductivity, $\mathrm{d}$ is the distance of interface from the plate $y=0, v$ is kinematic viscosity, $g$ the acceleration due to gravity, $\theta$ is the temperature at any point of the fluid flow. $L$ is the distance of vertical plates, $u$ the dynamic velocity, $U_{0}$ the velocity of the plate. $A$ is the plate temperature at $y=0$ and $B$ is the plate temperature at $y=1$. The subscripts $f$ represents a clear fluid layer, $p$ porous layer, $h$ hot plate and $c$ the cool plate.

Solutions:

It is observed that above governing equations are coupled non-linear. Accordingly, we assume for small but $(\ll 1)$, a very small Buoyancy parameter in most of the practical problems:

$$
\begin{aligned}
& u_{f}=u_{0 f}+\beta_{u} u_{1 f}+0\left(\beta u^{2}\right) \\
& u_{p}=u_{0 p}+\beta_{u} u_{1 p}+0\left(\beta u^{2}\right) \\
& \theta_{f}=\theta_{0 f}+\beta_{u} \theta_{1 f}+0\left(\beta u^{2}\right) \\
& \theta_{p}=\theta_{0 p}+\beta_{u} \theta_{1 p}+0\left(\beta u^{2}\right)
\end{aligned}
$$

Substituting the Equation (12) into the Equations (7)-(10), we have:

$$
\begin{aligned}
& \frac{\mathrm{d}^{2} u_{0 f}}{\mathrm{~d} y^{2}}-M^{2} u_{0 f}+\theta_{0 f}=0 \\
& \frac{\mathrm{d}^{2} u_{1 f}}{\mathrm{~d} y^{2}}-M^{2} u_{1 f}+\theta_{1 f}=0
\end{aligned}
$$




$$
\begin{gathered}
\frac{\mathrm{d}^{2} \theta_{0 f}}{\mathrm{~d} y^{2}}=0 \\
\frac{\mathrm{d}^{2} \theta_{1 f}}{\mathrm{~d} y^{2}}+\left(\frac{\mathrm{d} u_{0 f}}{\mathrm{~d} y}\right)^{2}+M^{2} u_{0 f}^{2}=0 \\
\frac{\mathrm{d}^{2} u_{0 p}}{\mathrm{~d} y^{2}}-M_{1}^{2} u_{0 p}+\theta_{0 p}=0 \\
\frac{\mathrm{d}^{2} u_{1 p}}{\mathrm{~d} y^{2}}-M_{1}^{2} u_{1 p}+\theta_{1 p}=0 \\
\frac{\mathrm{d}^{2} \theta_{0 p}}{\mathrm{~d} y^{2}}=0 \\
\frac{\mathrm{d}^{2} \theta_{1 p}}{\mathrm{~d} y^{2}}+\left(\frac{\mathrm{d} u_{0 p}}{\mathrm{~d} y}\right)^{2}+M_{1}^{2} u_{0 p}^{2}=0
\end{gathered}
$$

The corresponding boundary conditions are:

$$
\begin{aligned}
& y=0, u_{0 f}=\bigcup_{0}, u_{1 f}=0, \theta_{0 f}=A, \theta_{1 f}=0 \\
& y=1, u_{0 p}=-\bigcup_{0}, u_{1 p}=0, \theta_{0 p}=B, \theta_{1 p}=0 \\
& y=d, u_{0 f}=u_{0 p}, u_{1 f}=u_{1 p}, \frac{\mathrm{d} u_{0 f}}{\mathrm{~d} y}=\frac{\mathrm{d} u_{0 p}}{\mathrm{~d} y}, \frac{\mathrm{d} u_{1 f}}{\mathrm{~d} y}=\frac{\mathrm{d} u_{1 p}}{\mathrm{~d} y} \\
& \theta_{0 f}=\theta_{0 p}, \theta_{1 f}=\theta_{1 p}, \frac{\mathrm{d} \theta_{0 f}}{\mathrm{~d} y}=\frac{\mathrm{d} \theta_{0 p}}{\mathrm{~d} y}, \frac{\mathrm{d} \theta_{1 f}}{\mathrm{~d} y}=\frac{\mathrm{d} \theta_{1 p}}{\mathrm{~d} y}
\end{aligned}
$$

Solving Equations (15) and (19) using boundary conditions (21) gives the following temperature component:

$$
\theta_{0 f}=\theta_{0 p}=A+(B-A) y
$$

Now solving Equations (13) and (17) by the homotopy perturbation technique with boundary conditions (21), construct homotopy (He [34], [36] and Biazar [37]):

$$
\begin{aligned}
& H=L\left(u_{0 f}\right)-L\left(u_{0 f i}\right)+P\left[L\left(u_{0 f i}\right)+N\left(u_{0 f}\right)-f a j\right]=0 \\
& H=L\left(u_{0 p}\right)-L\left(u_{0 p i}\right)+P\left[L\left(u_{0 f i}\right)+N\left(u_{0 p}\right)-f a j\right]=0
\end{aligned}
$$

where $L\left(u_{0 f}\right), L\left(u_{0 p}\right)$ and $N\left(u_{0 f}\right), N\left(u_{0 p}\right)$ is the linear term of $u_{0 f}$ and $u_{0 p}$ and Non-Linear term of $u_{0 f}$ and $u_{0 p}$ respectively and $u_{0 f i}, u_{0 p i}$ is the initial value of $u_{0 f}, u_{0 p}$.

Let

$$
\begin{aligned}
& u_{0 f}=u_{00 f}+P u_{01 f}+O\left(P^{2}\right) \\
& u_{0 p}=u_{00 p}+P u_{01 p}+O\left(P^{2}\right)
\end{aligned}
$$

Boundaries and matching conditions are:

$$
\begin{gathered}
u_{0 f}=\bigcup_{0}, u_{00 f}=\bigcup_{0}, u_{01 f}=0 \text { at } y=0 \\
u_{0 p}=-\bigcup_{0}, u_{00 p}=-\bigcup_{0}, u_{01 p}=0 \text { at } y=1 \\
u_{0 f}=u_{0 p}, u_{00 f}=u_{00 p}, u_{01 f}=u_{01 p} \text { at } y=d
\end{gathered}
$$

And 


$$
\frac{\mathrm{d} u_{0 f}}{\mathrm{~d} y}=\frac{\mathrm{d} u_{0 p}}{\mathrm{~d} y}, \frac{\mathrm{d} u_{00 f}}{\mathrm{~d} y}=\frac{\mathrm{d} u_{00 p}}{\mathrm{~d} y}, \frac{\mathrm{d} u_{01 f}}{\mathrm{~d} y}=\frac{\mathrm{d} u_{01 p}}{\mathrm{~d} y} \text { at } y=d
$$

Then the solution of Equations (13) and (17):

$$
\begin{aligned}
& u_{0 f}=C_{1} \mathrm{e}^{M y}+C_{2} \mathrm{e}^{-M y}+\frac{A+(B-A) y}{M^{2}} \\
& u_{0 p}=C_{3} \mathrm{e}^{M_{1} y}+C_{4} \mathrm{e}^{-M_{1} y}+\frac{A+(B-A) y}{M_{1}^{2}}
\end{aligned}
$$

With the help of a solution of Equations (22), (27) and (28), (29), we get the solution of Equations (14), (16), (18), and (20):

$$
\begin{aligned}
\theta_{1 f}= & C_{5} y+C_{6}+A_{1} \mathrm{e}^{2 M y}+A_{2} \mathrm{e}^{-2 M y}+A_{3} y^{2}+A_{4} \mathrm{e}^{M y}+A_{5} \mathrm{e}^{-M y} \\
& +A_{6} y^{4}+A_{7} \frac{y^{3}}{6}+A_{8} \mathrm{e}^{M y}\left(y-\frac{2}{M}\right)+A_{9} \mathrm{e}^{-M y}\left(y+\frac{2}{M}\right) \\
\theta_{1 p}= & C_{7} y+C_{8}+B_{1} \mathrm{e}^{2 M_{1} y}+B_{2} \mathrm{e}^{-2 M_{1} y}+B_{3} y^{2}+B_{4} \mathrm{e}^{M_{1} y}+B_{5} \mathrm{e}^{-M_{1} y} \\
& +B_{6} y^{4}+B_{7} \frac{y^{3}}{6}+B_{8} \mathrm{e}^{M_{1} y}\left(y-\frac{2}{M_{1}}\right)+B_{9} \mathrm{e}^{-M_{1} y}\left(y+\frac{2}{M_{1}}\right)
\end{aligned}
$$

And

$$
\begin{aligned}
u_{1 f}= & C_{9} \mathrm{e}^{M y}+C_{10} \mathrm{e}^{-M y}+A_{10} \mathrm{e}^{2 M y}+A_{11} \mathrm{e}^{-2 M y}+A_{12}\left(y^{2}+\frac{2}{M^{2}}\right)+A_{13} y \mathrm{e}^{M y} \\
& +A_{14} y \mathrm{e}^{-M y}+A_{15}\left(y^{4}+\frac{12}{M^{2}} y^{2}+\frac{24}{M^{4}}\right)+A_{16}\left(y^{3}+\frac{6 y}{M^{2}}\right) \\
& +A_{17}\left(\frac{y^{2}}{4 M}-\frac{5 y}{4 M^{2}}\right) \mathrm{e}^{M y}+A_{18}\left(\frac{y^{2}}{4 M}+\frac{5 y}{4 M^{2}}\right) \mathrm{e}^{-M y}+\frac{C_{5} y}{M^{2}}+\frac{C_{6}}{M^{2}} \\
u_{1 p}= & C_{11} \mathrm{e}^{M_{1} y}+C_{12} \mathrm{e}^{-M_{1} y}+B_{10} \mathrm{e}^{2 M_{1} y}+B_{11} \mathrm{e}^{-2 M_{1} y}+B_{12}\left(y^{2}+\frac{2}{M_{1}^{2}}\right)+B_{13} y \mathrm{e}^{M_{1} y} \\
+ & B_{14} y \mathrm{e}^{-M_{1} y}+B_{15}\left(y^{4}+\frac{12}{M_{1}^{2}} y^{2}+\frac{24}{M_{1}^{4}}\right)+B_{16}\left(y^{3}+\frac{6 y}{M_{1}^{2}}\right) \\
+ & B_{17}\left(\frac{y^{2}}{4 M_{1}}-\frac{5 y}{4 M_{1}^{2}}\right) \mathrm{e}^{M_{1} y}+B_{18}\left(\frac{y^{2}}{4 M_{1}}+\frac{5 y}{4 M_{1}^{2}}\right) \mathrm{e}^{-M_{1} y}+\frac{C_{7} y}{M_{1}^{2}}+\frac{C_{8}}{M_{1}^{2}}
\end{aligned}
$$

The constants are dropped for the sake of brevity.

\section{Skin Friction Coefficient}

The numerical values of Skin-friction are exposed in Table 1 for $U_{0}=0.4 . \tau_{1}, \tau_{2}$ is the skin friction when $A=1$, $B=0$ and $A=0, B=1$ respectively. It is evident from Table that getting bigger the width of the clear fluid layer increases the skin friction. It is also analyzed that skin friction on both the plates increases when those are heated. The influence of Hartmann number $(M)$ is to suppress the skin friction for clear fluid region and is to enhance the skin friction for porous medium.

\section{Result and Discussion}

In order to mull over the configuration of magneto-hydrodynamic two-phase flow in vertical plates, channels partially filled with porous substrate when plates are moving in opposite directions has been discussed. The closed form of solutions of nonlinear-coupled momentum and energy equations have been analytically obtained by the homotopy perturbation technique and the solutions of ordinary differential equation method. The findings 
Table 1. Skin friction coefficient.

\begin{tabular}{|c|c|c|c|c|c|c|c|}
\hline \multirow{3}{*}{$\beta u$} & \multirow{3}{*}{ M } & \multirow{3}{*}{$D a$} & \multirow{3}{*}{ d } & $A=1$ & $B=0$ & $A=0$ & $B=1$ \\
\hline & & & & \multicolumn{2}{|c|}{$\tau_{1}$} & \multicolumn{2}{|c|}{$\tau_{2}$} \\
\hline & & & & $u_{f}$ & $u_{p}$ & $u_{f}$ & $u_{p}$ \\
\hline \multirow{2}{*}{ 0 } & \multirow{2}{*}{1} & \multirow{2}{*}{0.1} & 0.5 & -0.5013 & 1.5671 & -0.6714 & 1.7053 \\
\hline & & & 0.7 & -0.4909 & 1.5702 & -0.6591 & 1.7116 \\
\hline \multirow{2}{*}{$\mathbf{0}$} & \multirow{2}{*}{1.5} & \multirow{2}{*}{0.1} & 0.5 & -0.6078 & 1.6118 & -0.7738 & 1.7485 \\
\hline & & & 0.7 & -0.6013 & 1.6613 & -0.7655 & 1.7509 \\
\hline \multirow{2}{*}{$\mathbf{0}$} & \multirow{2}{*}{1} & \multirow{2}{*}{0.01} & 0.5 & -0.5170 & 4.0395 & -0.6908 & 4.1185 \\
\hline & & & 0.7 & -0.3656 & 4.0741 & -0.5442 & 4.1529 \\
\hline \multirow{2}{*}{$\mathbf{0}$} & \multirow{2}{*}{1.5} & \multirow{2}{*}{0.01} & 0.5 & -0.6167 & 4.0633 & -0.7862 & 4.1420 \\
\hline & & & 0.7 & -0.4973 & 4.0928 & -0.6644 & 4.1715 \\
\hline \multirow{2}{*}{0.1} & \multirow{2}{*}{1} & \multirow{2}{*}{0.1} & 0.5 & -0.4906 & 1.5684 & -0.6565 & 1.7035 \\
\hline & & & 0.7 & -0.4789 & 1.5717 & -0.6411 & 1.7097 \\
\hline \multirow[b]{2}{*}{0.1} & \multirow[b]{2}{*}{1.5} & \multirow[b]{2}{*}{0.1} & 0.5 & -0.6060 & 1.6139 & -0.7654 & 1.7495 \\
\hline & & & 0.7 & -0.5993 & 1.6236 & -0.7566 & 1.7521 \\
\hline \multirow[b]{2}{*}{0.1} & \multirow[b]{2}{*}{1} & \multirow[b]{2}{*}{0.01} & 0.5 & -0.5063 & 4.0400 & -0.6724 & 4.1190 \\
\hline & & & 0.7 & -0.3514 & 4.0746 & -0.5256 & 4.1534 \\
\hline \multirow{2}{*}{0.1} & \multirow{2}{*}{1.5} & \multirow{2}{*}{0.01} & 0.5 & -0.6157 & 4.0638 & -0.7779 & 4.1425 \\
\hline & & & 0.7 & -0.4898 & 4.0934 & -0.6565 & 4.1721 \\
\hline
\end{tabular}

are presented graphically and discussed in detail. The velocity distribution (zeroth and first order) and temperature distribution (only first order) are depicted in the figures. The zeroth order temperature profile is not shown since it is linear. The effect of Darcy dissipation and joule dissipation is taken into account. There domains are viewed such as clear fluid domain (near $y=0$ ), interface domain (at $y=\mathrm{d}$ ) and pore domain (near the plate $y=$ 1).

The zeroth order velocity profile flow is plotted in Figure 1 and Figure 2. It is seen that with the increasing Darcy number $(D a)$ the flow velocity (magnitude) increases in the clear fluid region and shows the same effect in the pore region, which is perfectly attached to the plate moving in opposite directions. It is so for the reason that Darcian drag force in the attached porous substrate impedance is reduced and thus the flow is accelerated in it. The zeroth order velocity shows the constant nature near the interface in porous media for low Darcy number ( $D a=0.001)$ and the effect of Brinkman term is almost none so classical Darcy law is dominated. It is important to note that the velocity does not depict the constant nature of the interface with moderately increasing value of Darcy number ( $D a=0.01)$. Figure 1 and Figure 2 also exhibit the fluid velocity at the hand of the clear fluid heated plate $(y=0, A=1, B=0)$ is more than the cold plate $(y=0, A=0, B=1)$. On the other hand, it is said that the fluid velocity near of porous heated plate $(y=1, A=0, B=1)$ is less than the cold plate $(y=1, A=0, B$ $=0$ ). It is due to considerable fact that the temperature coupled with resistance offered by the porous matrix.

Figure 1 displays the effect of magnetic parameter $(M)$ on the velocity (magnitude) field, the figure makes known commonly that the velocity decreases with increasing $M$, signifies the increases of resistive type force (Lorentz force) which tends to retard the fluid flow velocity in the clear fluid region, interface, and porous region. It is noteworthy to the outlook that magnetic interaction with low Darcy parameter $(D a=0.001)$ does not contribute a lot to affect fluid flow in porous media. Figure 3 depicts the variation in zeroth order fluid velocity with variable magnetic field on a scale Darcy number ( $D a=0.01$ and $D a=0.001$, when $d=0$ ) for only porous fluid domain. 


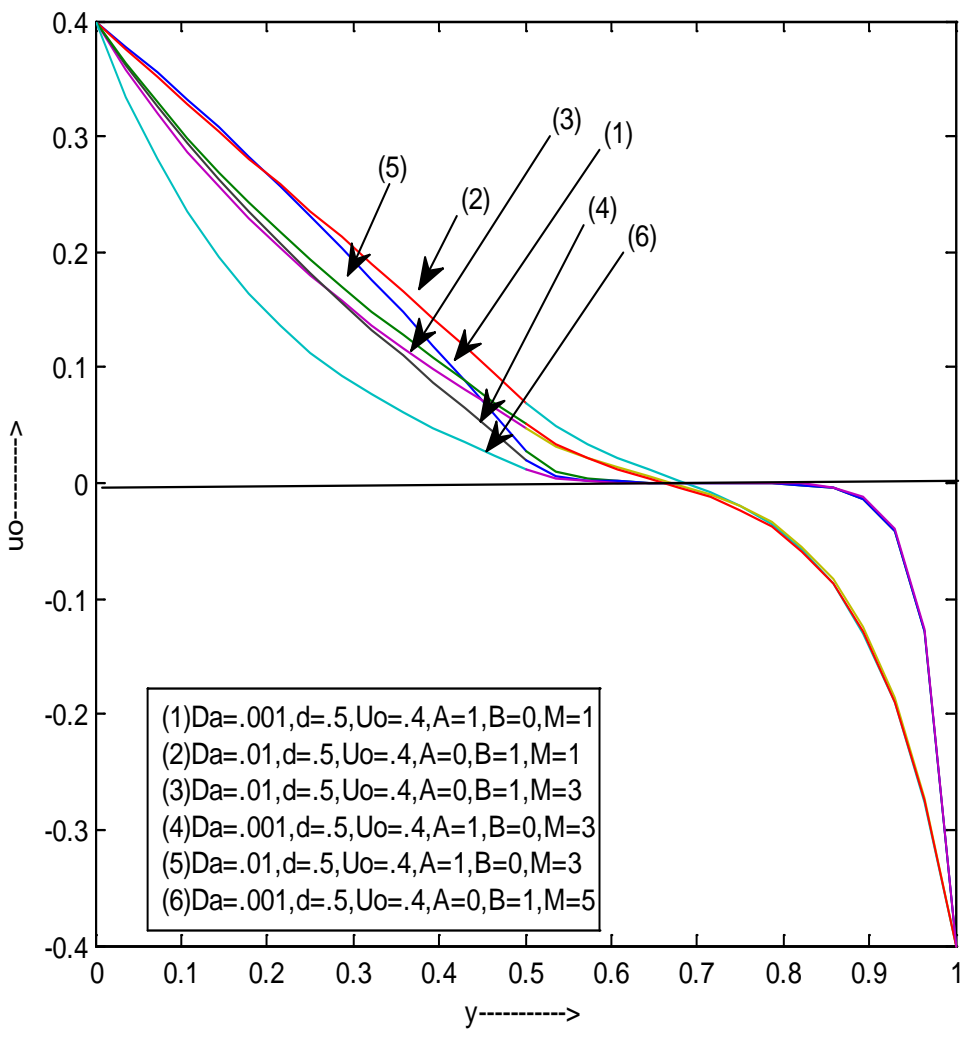

Figure 1. Graph of zeroth order velocity $U_{0}$ against $y$.

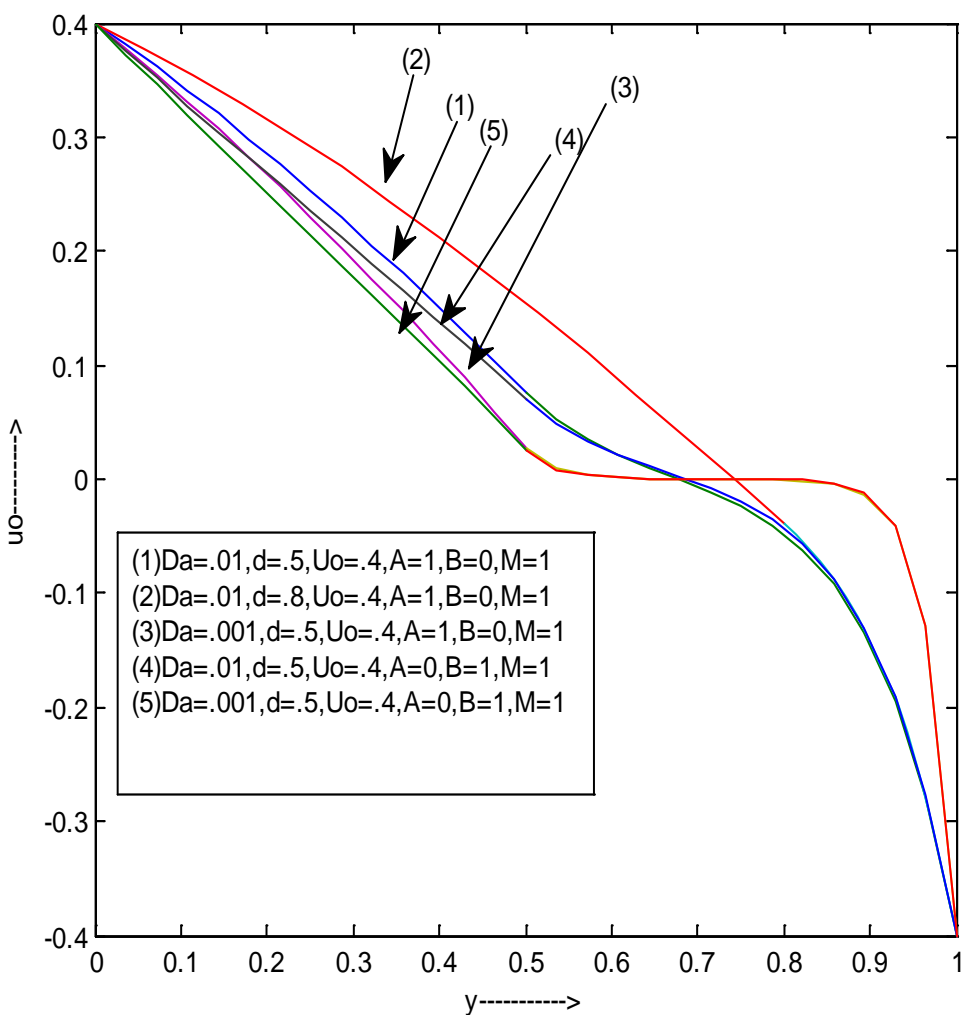

Figure 2. Zeroth order velocity against $y$. 


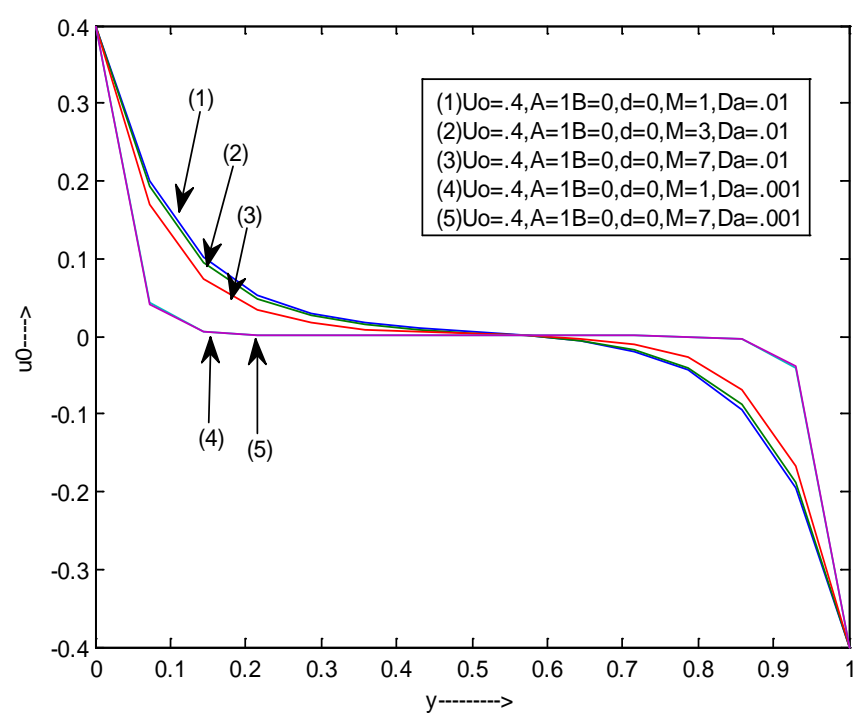

Figure 3. Zeroth order velocity against $y$.

Figure 4 of first order velocity portrays the dependence of first order velocity with the distance $y$ between the plates that shows the very small effect of first order velocity because of its nonlinear term existence. Fluid velocity increases with distance y and get maximum velocity in between $y=0$ to $y=\mathrm{d}$ for clear fluid flow region. The reason for this behavior is free flow of liquid between in the plate $(y=0)$ and interface (clear-porous $y=\mathrm{d}$ ) and the fluid is in contact with the interface boundary (clear-porous $y=\mathrm{d}$ ) which induces the resistive effect at the boundary. The fluid motion is not free due to the presence of the porous substrate between $y=\mathrm{d}$ to $y=1$. The obstacles due to porosity reverse the fluid velocity which causes the fluid velocity decreases to attain very small value at $y=1$. It may be noted that decreasing the Darcy number the fluid velocity decreases and attains a very low velocity, which is also acceptable, physically in very small value of Darcy number $(D a=0.001)$. Figure 5 depicts the variation in first order fluid velocity on a very small scale Darcy number ( $D a=0.001$ ). Figure 4 also shows that increasing the strength of the magnetic field the decay of fluid flow is greater in all regions. It is so because the retarding effect of magnetic field.

Figure 6 and Figure 7 illustrate the similar behavior of temperature dependence against $y$ by changing the width of free fluid flow in the region $y=0$ to $y=\mathrm{d}$ and in porous media from $y=d$ to $y=1$. These figures also indicate that temperature of liquid in the free flow region is maximum when $\mathrm{d}$ is small and reverse behavior can be noted in a porous substrate for both of the Darcy parameters $D a=0.01$ and $D a=0.001$.

Figure 8 shows the temperature variation of liquid with distance $y$ between the plates when plates $A$ and $B$ are heated. It is showing a sign of the temperature distribution of fluid decreases when near the plate of porous matrix is heated. This is due to the fact that heat supplied by plate $B$ attached to the porous regime is absorbed by the porous substrate and less transition of heat flow between the plates giving decline the temperature profile in all regions. Similar behavior is shown in Figure 9 for $D a=0.001$.

Figure 10 and Figure 11 depict the similar behavior of variation of fluid temperature against the distance $y$. It is noted that by decreasing the permeability of porous substrate the temperature profile increases. These figures conclude the considerable effect of non-linear term, and signify the viscous and Darcy dissipation. It is interesting to conclude that temperature difference profile between the plates become more or less flat over most of the plates because the very less transition of temperature between the plates giving mount thermal boundary layer flow adjacent to both of the plates for very low Darcy parameter like 0.001 which leads to viscous dissipation and Darcy dissipation. The flattening in temperature variation is due to decreases in Darcy parameter, which causes the Darcy drag force.

Figure 12 reveals the variation of the temperature profile of liquid with the distance between the plate and indicates that as the velocity of plates are increasing the Darcy dissipation increases which causes increases the temperature profile of fluid.

Figures 13-15 represent the temperature profile in the boundary layer and momentum of various value of Hartmann number $(M)$. It is encountered from these figures that temperature distribution increases in thermal 


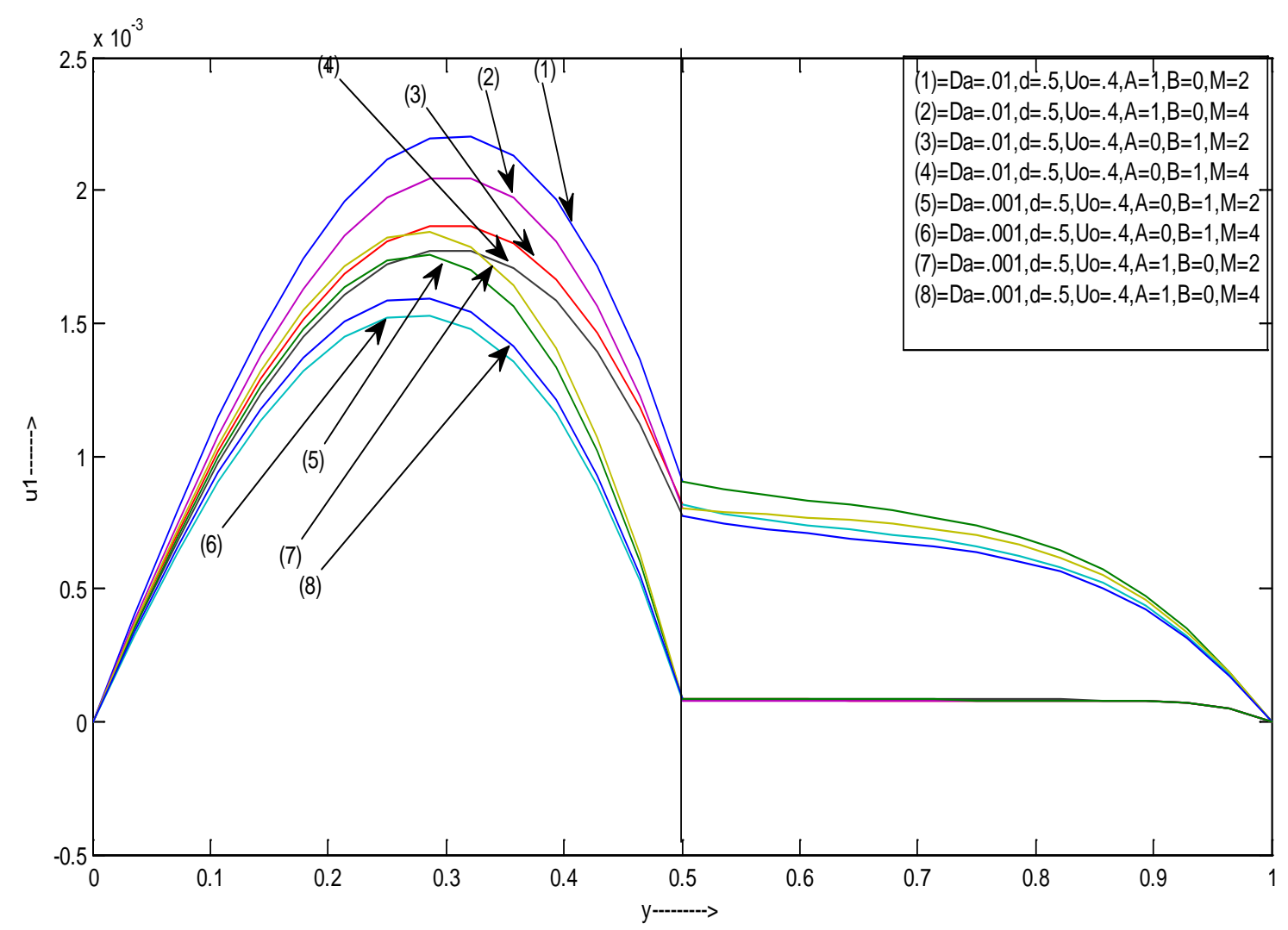

Figure 4. First order velocity against $v$.

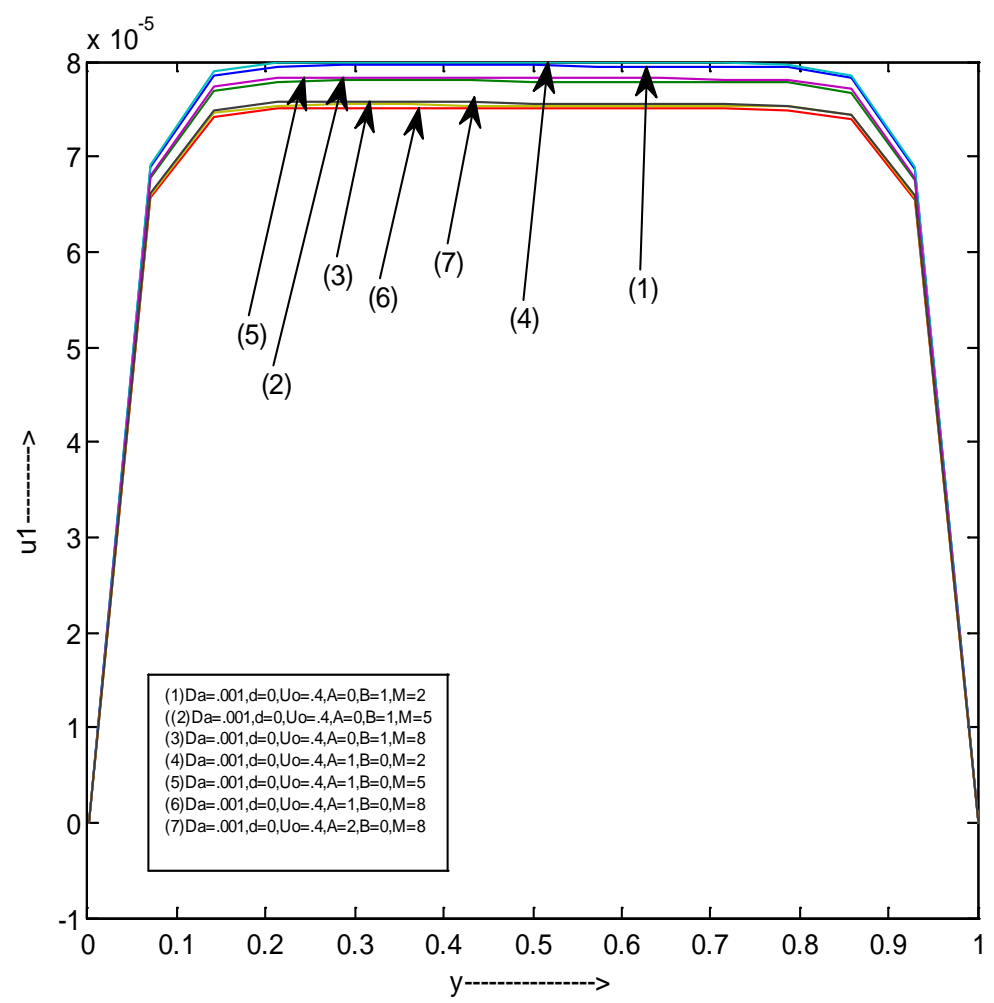

Figure 5. First order velocity against $y$. 


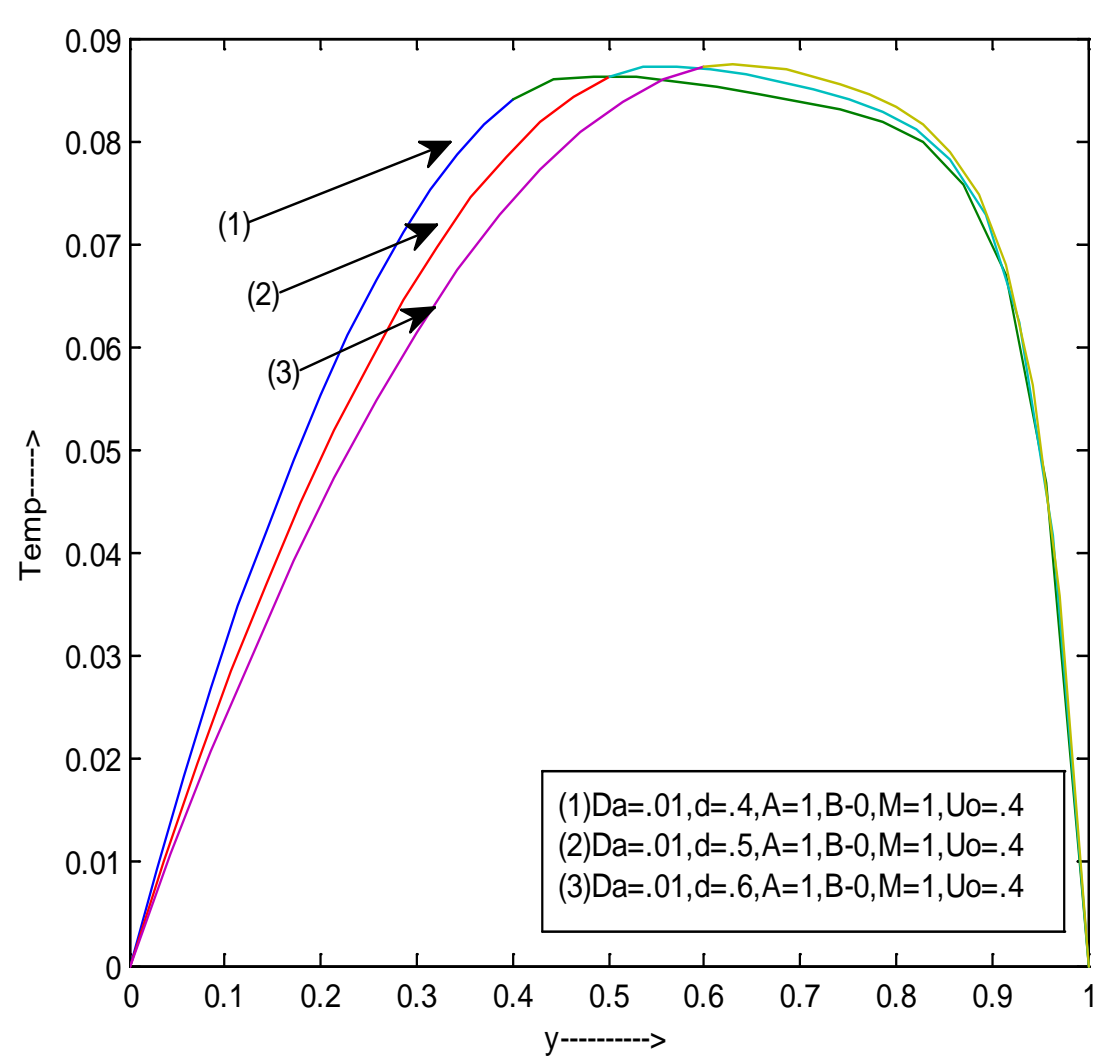

Figure 6. Temperature against $y$.

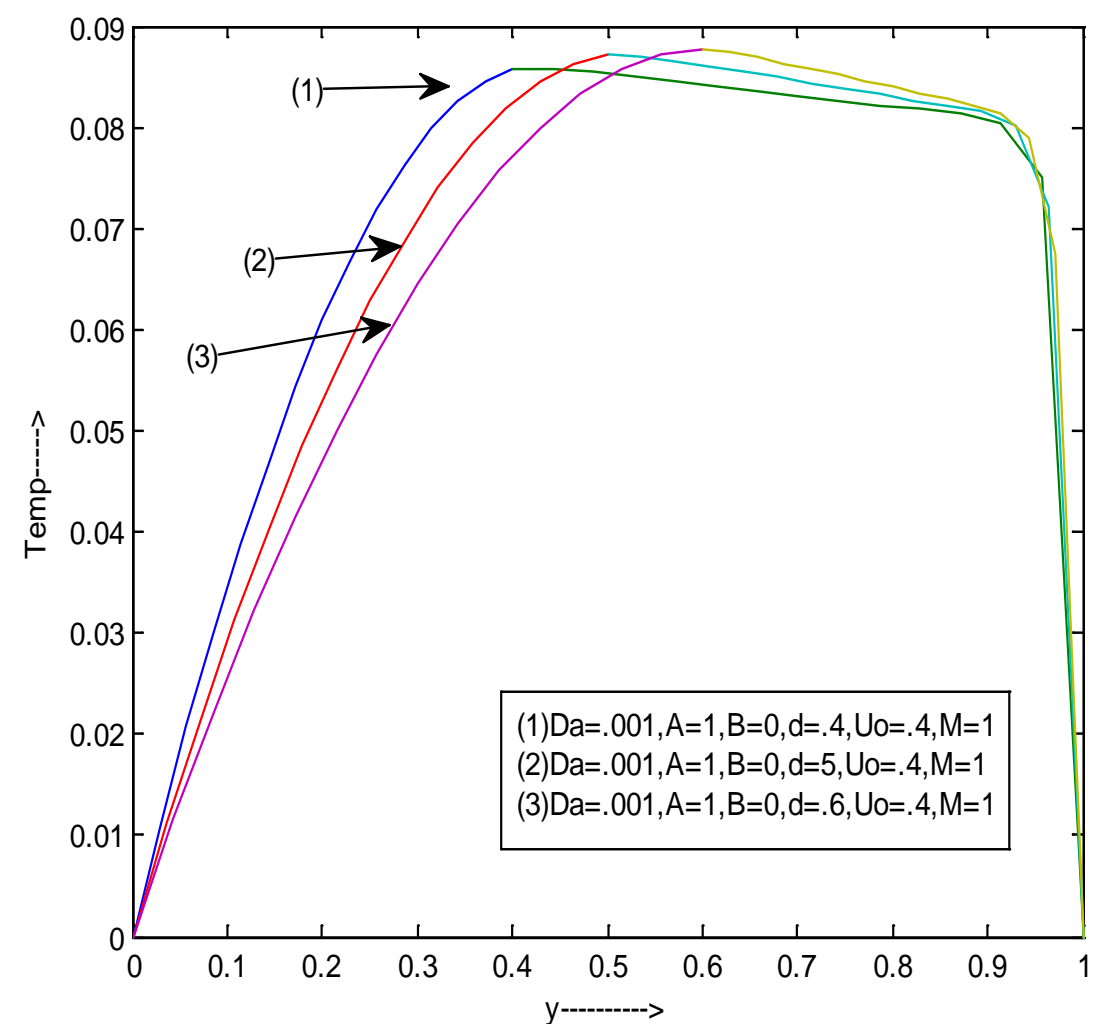

Figure 7. Temperature against $y$. 


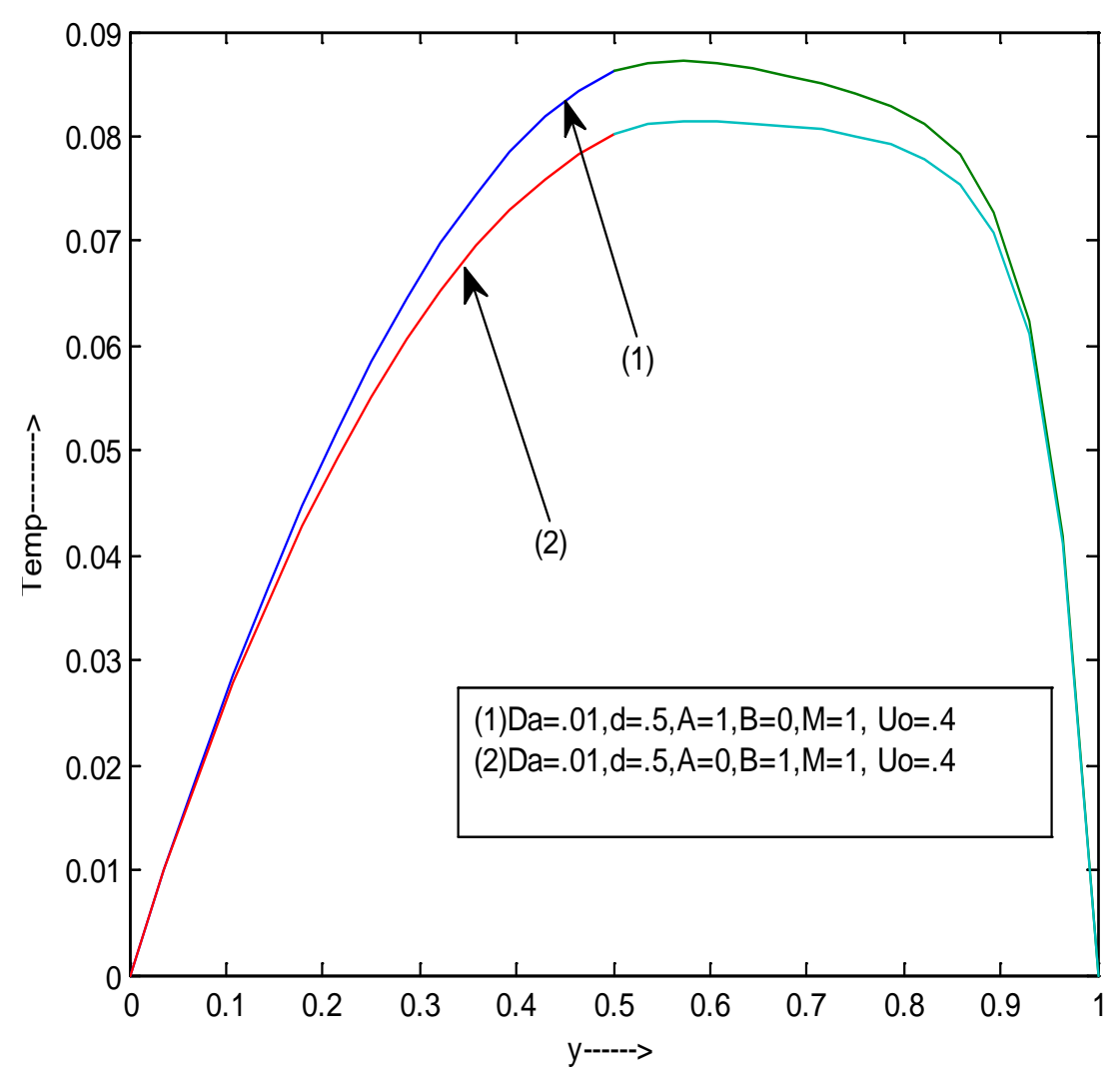

Figure 8. Temperature against $y$.

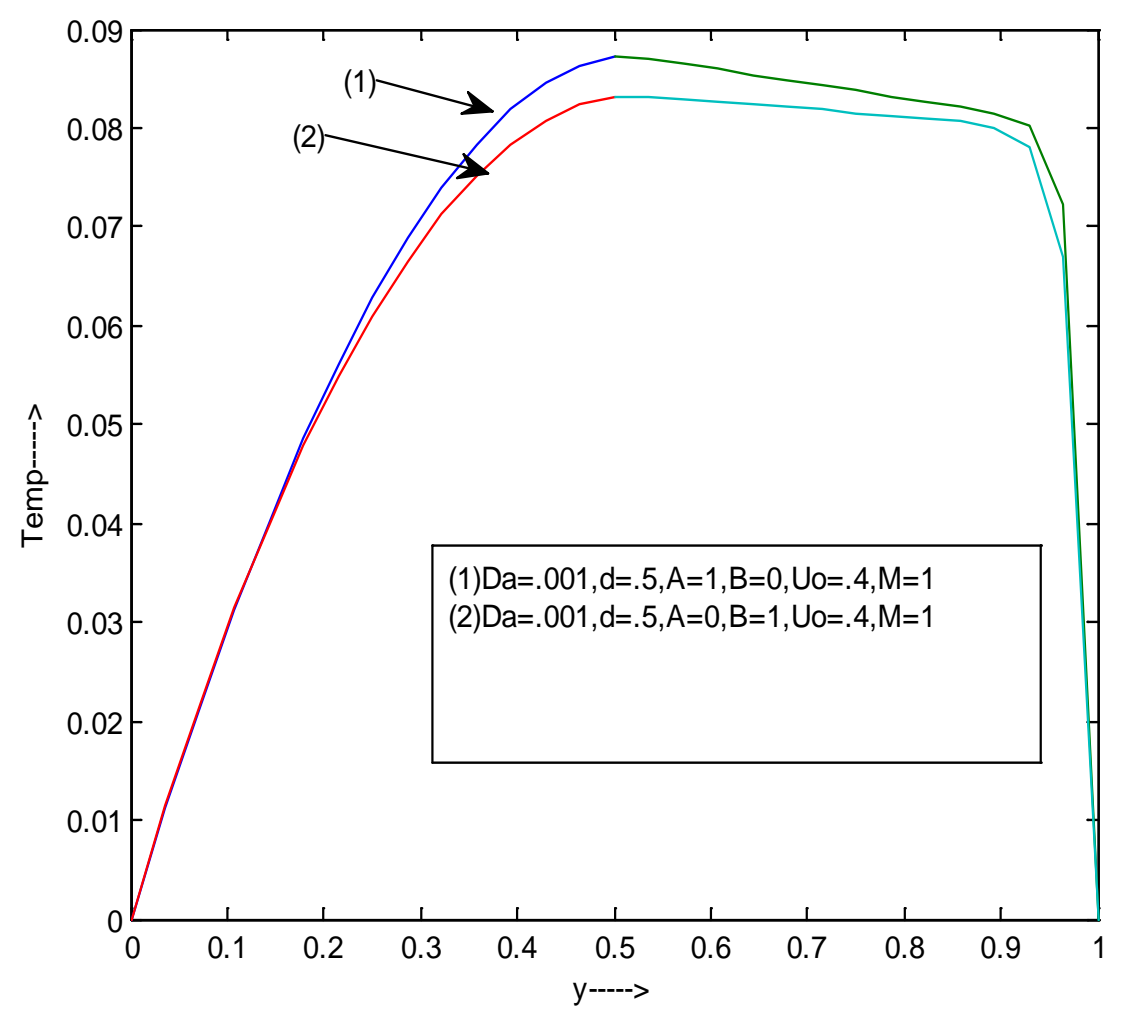

Figure 9. Temperature against $y$. 


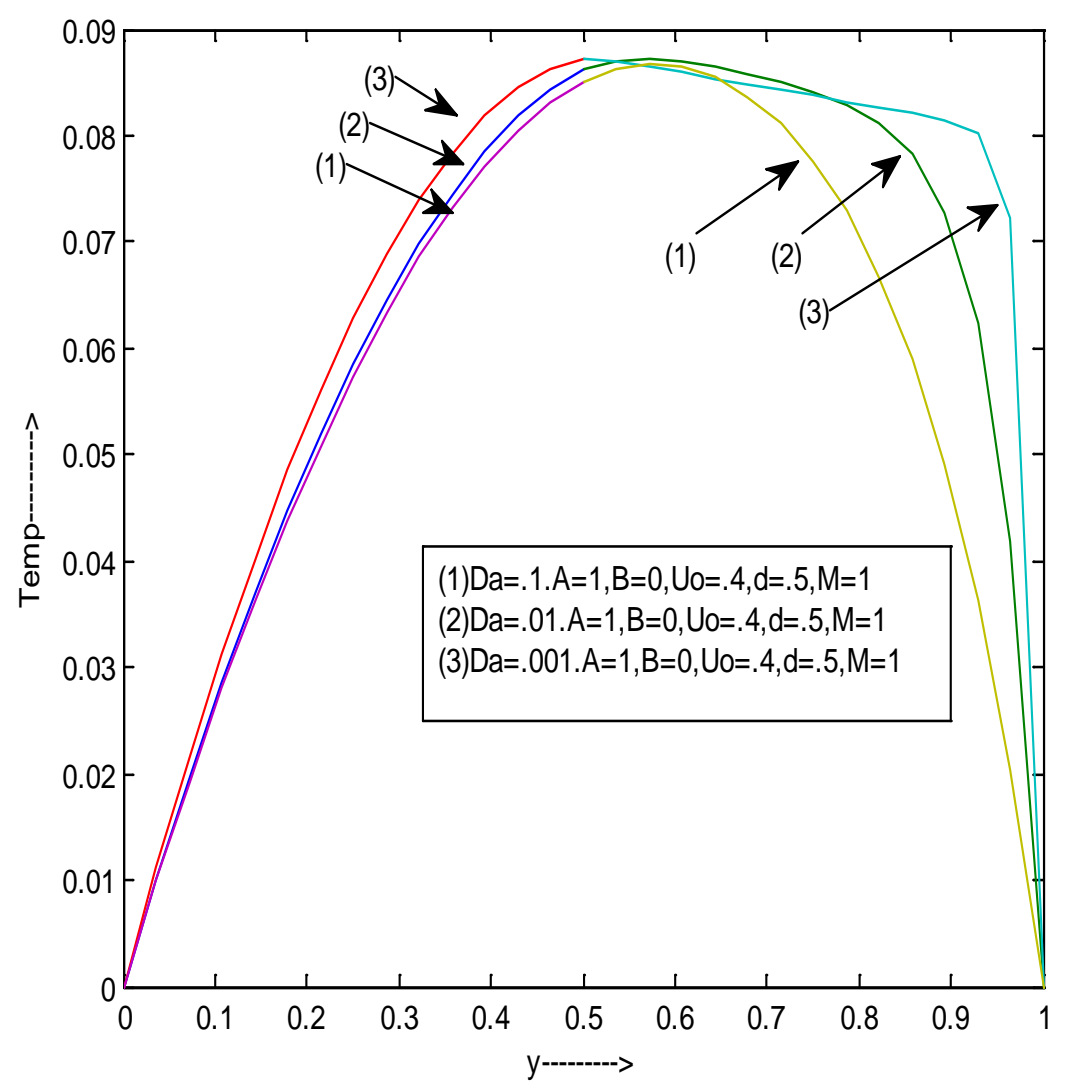

Figure 10. Temperature against $y$.

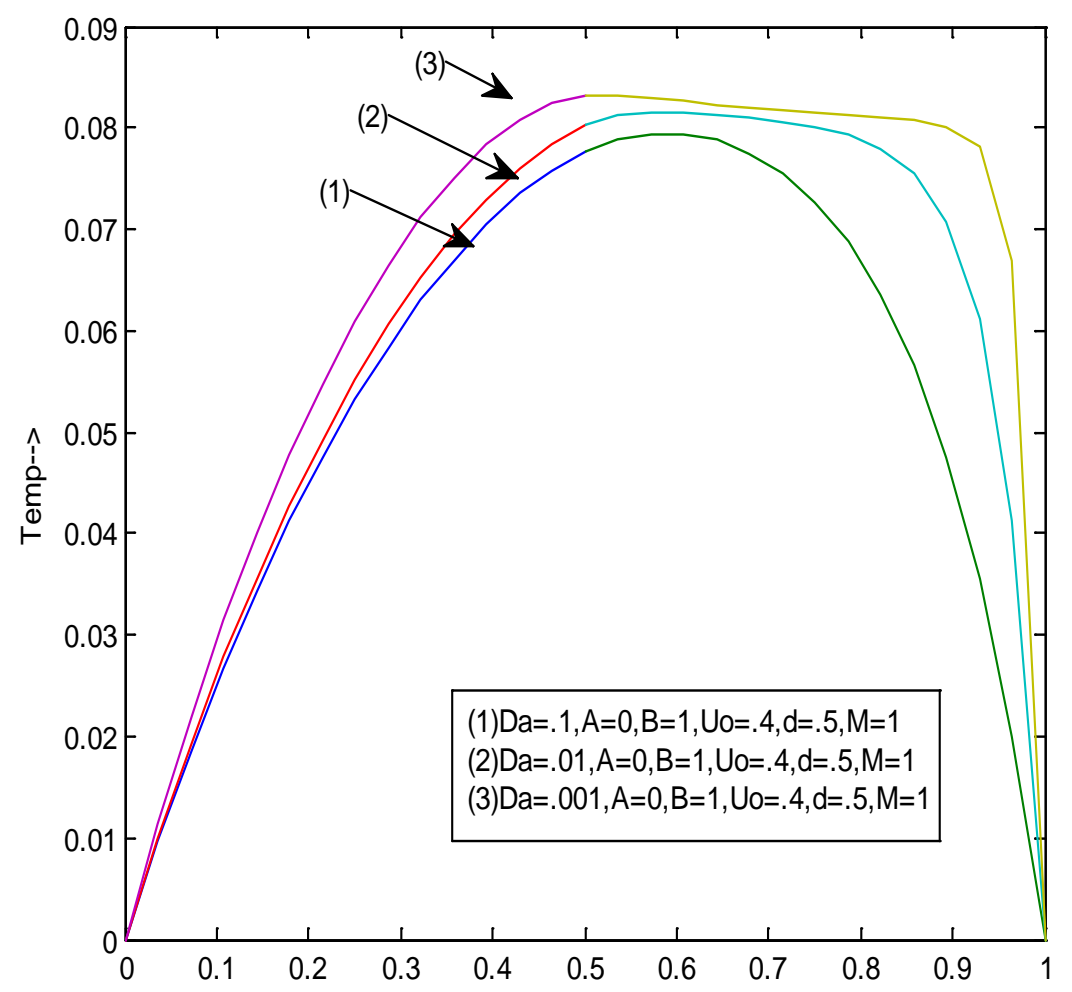

Figure 11. Temperature against $y$. 


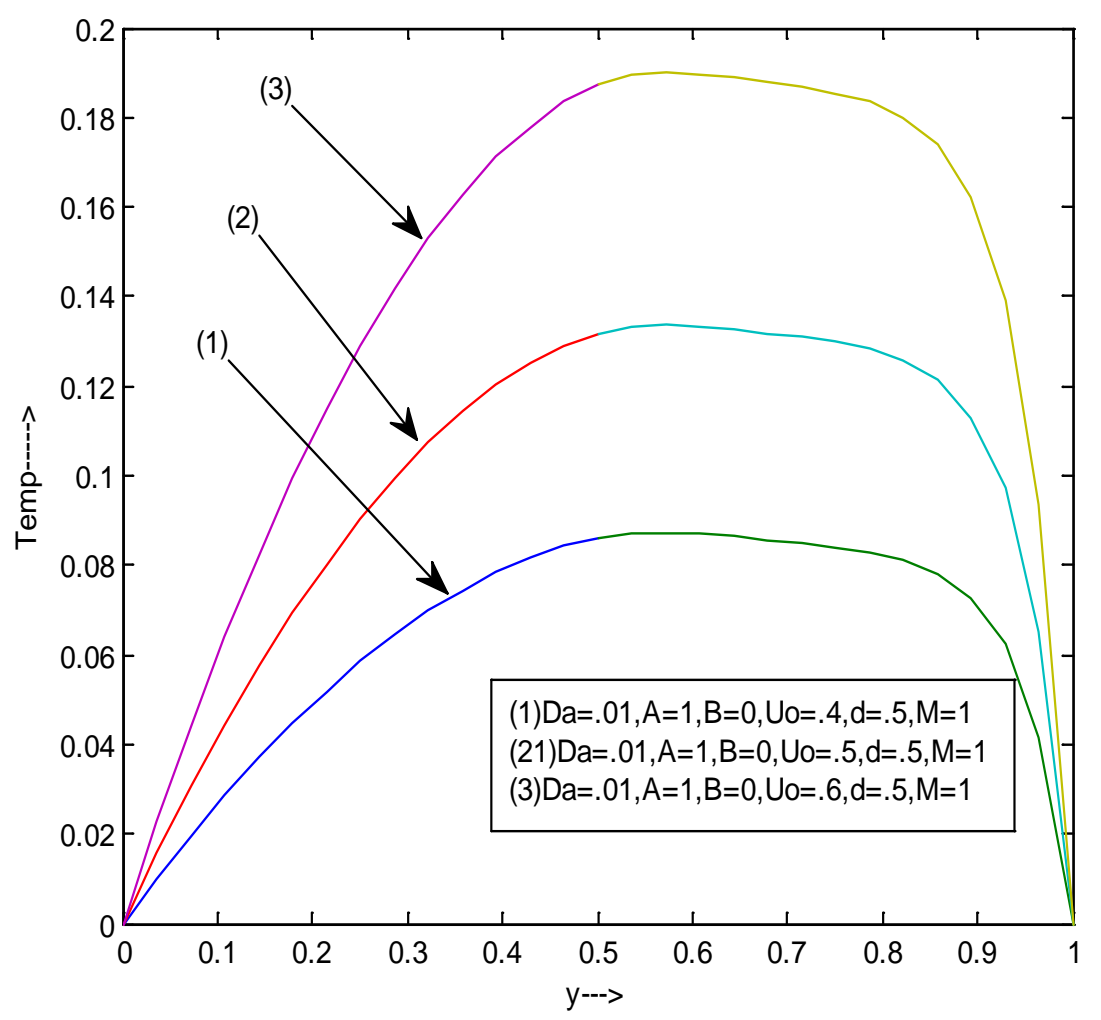

Figure 12. Temperature against $y$.

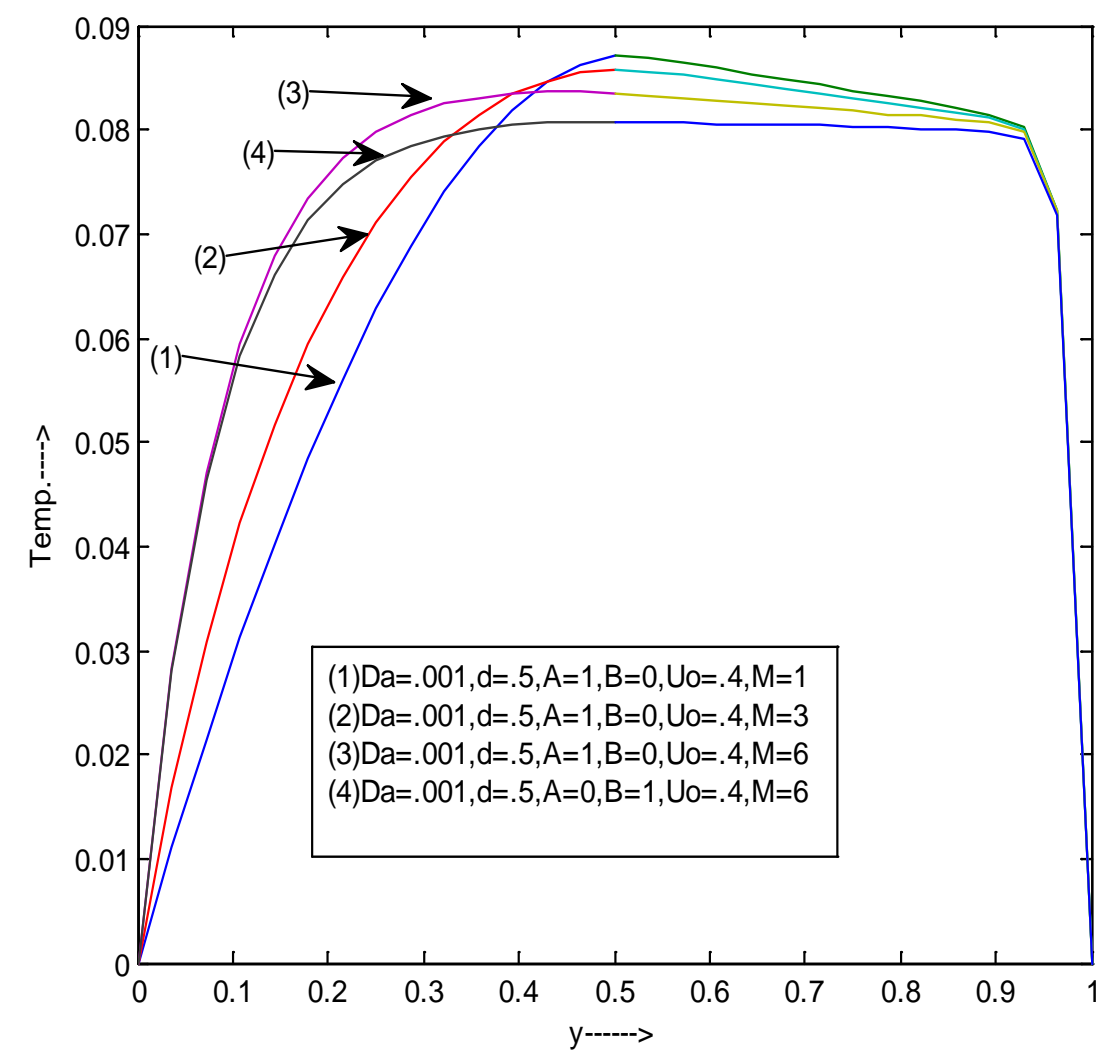

Figure 13. Temperature against $y$. 


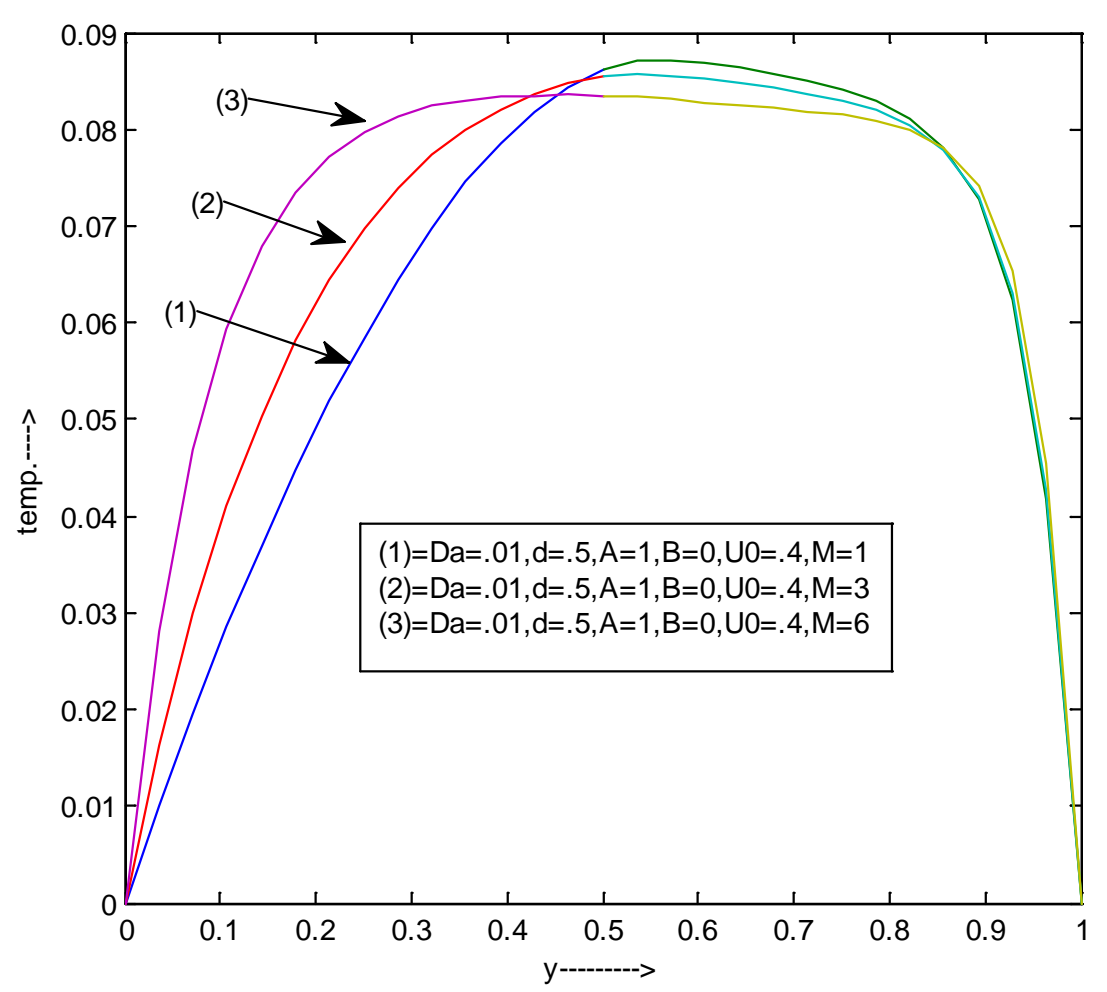

Figure 14. Temperature against $y$.

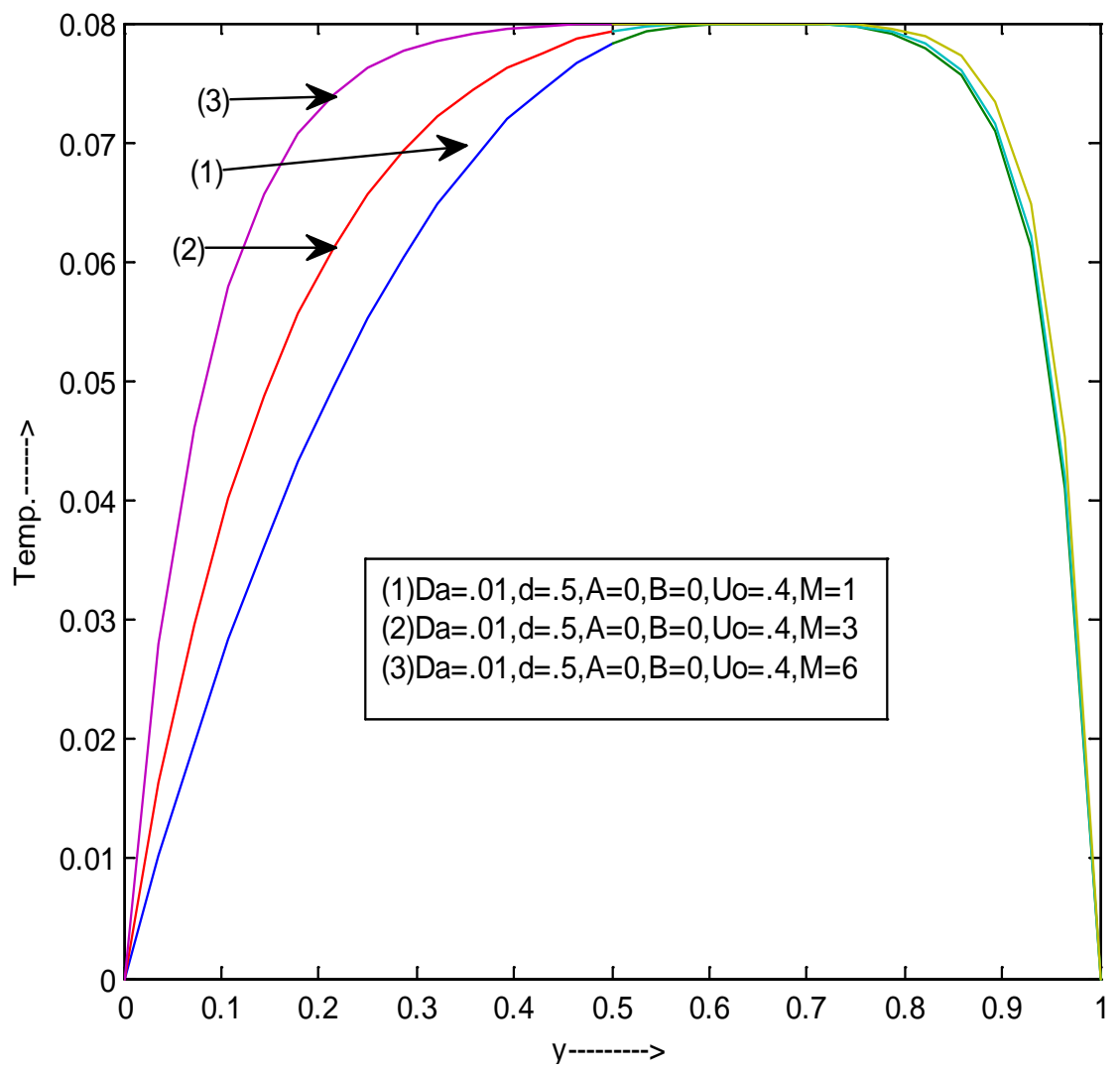

Figure 15. Temperature against $y$. 
boundary layer adjacent to the plates with growing the Hartmann number $(M)$ for both of the Darcy parameters $(0.01,0.001)$. It is attributed to the fact that kinetic energy lost from the fluid flow due to the magnetic field effect is manifested as joule (ohmic) heating so Hartmann contributed significantly in generation of temperature. Temperature profile remains almost constant with increasing the value of Hartmann number $(M)$ in porous media and it is greater in the clear fluid in close proximity to the plate. Therefore, it is imperative to conclude that magnetic parameter associated with Darcy parameter fails to contribute much temperature distribution in momentum. It is interesting to note of these figures that giving raises to temperature in the flow field either A or B exists.

\section{Conclusions}

1) Presence of magnetic field $(M)$ decreases the velocity (magnitude) of flow field in the clear and porous fluid region. It is so as a retarding effect of Lorentz force.

2) Velocity (magnitude) of fluid falls in whole region with decreasing Darcy parameter (Da).

3) Temperature coupled with Darcy parameter decelerates the motion flow and decreases the temperature profile of fluid.

4) Inclusion of magnetic field with Darcy parameter is the beneficial gaining temperature in the thermal boundary layer adjacent to the plates.

5) Low Darcy number enhances the temperature distribution near the plates.

6) Shear stress on both of the plates $(y=0$ and $y=1)$ increases due to increasing the width of the clear fluid layer.

7) The skin friction decreases with the increasing Hartmann number $(M)$ in clear fluid, but the reversal effect shows in a porous medium and skin friction on both the plates ( comparing at $y=0$ and at $y=1$ for $A$ or $B$ ) increases when those are heated.

8) Giving raises to temperature in the flow field either $A$ or $B$ exists.

\section{Acknowledgements}

Author Ajay Jain is thankful to Prof (Dr.) R.S. Tiwari Director (R \& D), RCERT for valuable suggestions and discussion for improvement of manuscript of the paper.

Author Ajay Jain is thankful to Referee for valuable suggestions.

\section{References}

[1] Hartmann, J. (1937) Theory of the Laminar Flow of an Electrically Conductive Liquid in a Homogeneous Magnetic Field. Fys. Med.., 15, 1-27.

[2] Rossow, V.J. (1957) Impulsive Motion of an Infinite Flat Plate in a Hydro-Magnetic Viscous Incompressible Fluid. Vol. 3, NACA-TN, Report, 489-508.

[3] Helliwell, J.B. (1977) On the Stability of Thermally Radiative Magneto Fluid Dynamic Channel Flow. Journal of Engineering Mathematics, 11, 67-80. http://dx.doi.org/10.1007/BF01535588

[4] Attia, H.A. and Kotb, N.A. (1996) MHD Flow between Two Parallel Plates with Heat Transfer. Acta Mechanic, 117, 215-220. http://dx.doi.org/10.1007/BF01181049

[5] Malathy, T. and Srinivas, S. (2008) Pulsating Flow of a Hydro-Magnetic Fluid between Permeable Beds. International Communications in Heat and Mass Transfer, 35, 681-688. http://dx.doi.org/10.1016/j.icheatmasstransfer.2007.12.006

[6] Singh, R.K., Singh, A.K., Sacheti, N.C. and Chandran, P. (2010) On Hydromagnetic Free Convection in the Presence of Induced Magnetic Field. Heat and Mass Transfer, 47, 523-529. http://dx.doi.org/10.1007/s00231-010-0594-6

[7] Beavers, G.S. and Joseph, D.D. (1967) Boundary Conditions at a Naturally Permeable Wall. Journal of Fluid Mechanics, 30, 197-207. http://dx.doi.org/10.1017/S0022112067001375

[8] Taylor, G.I. (1971) A Model for Boundary Conditions of a Porous Material—Part 1. Journal of Fluid Mechanics, 49, 319-326. http://dx.doi.org/10.1017/S0022112071002088

[9] Richardson, S. (1971) A Model for the Boundary Condition of a Porous Material—Part 2. Journal of Fluid Mechanics, 49, 327-336. http://dx.doi.org/10.1017/S002211207100209X

[10] Degen, M.M., Colovas, P.W. and Andereck, C.D. (1988) Time Dependent Patterns in the Two-Layer Rayleigh-Benard System. Physics Review E, 57, 6647-6659. http://dx.doi.org/10.1103/PhysRevE.57.6647 
[11] Lohrasbi, J. and Sahai, V. (1988) Magnetohydrodynamic Heat Transfer in Two Phase Flow between Parallel Plates. Applied Science Research, 45, 53-66. http://dx.doi.org/10.1007/BF00384182

[12] Vafai, K. and Kim, S.J. (1995) On the Limitation of the Brinkman-Forchheimer-Extended Darcy Equation. International Journal of Heat Fluid Flow, 16, 11-15. http://dx.doi.org/10.1016/0142-727X(94)00002-T

[13] Vafai, K. and Kim, S.J. (1990) Fluid Mechanics of the Interface Region between a Porous Medium and a Fluid Layer: An Exact Solution. International Journal of Heat Fluid Flow, 11, 254-256. http://dx.doi.org/10.1016/0142-727X(90)90045-D

[14] Kuznetsov, A.V. (1998) Thermal Non-Equilibrium Forced Convection in Porous Media. In: Inghan, D.B. and Pop, I., Eds., Transport Phenomena in Porous Media, Elsevier, Oxford, 103-129. http://dx.doi.org/10.1016/B978-008042843-7/50005-2

[15] Alazmi, B. and Vafai, K. (2001) Analysis of Fluid Flow and Heat Transfer Interfacial Conditions between a Porous Medium and a Fluid Layer. International Journal of Heat Mass Transfer, 44, 1735-1749. http://dx.doi.org/10.1016/S0017-9310(00)00217-9

[16] Valencia-Lopez, J.J. and Ochoa Tapia, J.A. (2001) A Study of Buoyancy-Driven Flow in a Confined Fluid Overlying a Porous Layer. International Journal of Heat Mass Transfer, 44, 4725-4736. http://dx.doi.org/10.1016/S0017-9310(01)00105-3

[17] Malashetty, M.S. and Leela, V. (1992) Magnetohydrodynamic Heat Transfer in Two Phase Flow. International Journal of Engineering Science, 30, 371-377. http://dx.doi.org/10.1016/0020-7225(92)90082-R

[18] Umavathi, J.C., Malashetty, M.S. and Mateen, A. (2004) Fully Developed Flow and Heat Transfer in a Horizontal Channel Containing Electrically Conducting Fluid Sandwiched between Two Fluid Layers. International Journal of Applied Mechanics and Engineering, 9, 781-794.

[19] Umavathi, J.C., Chamkha, A.J., Mateen, A. and Mudhaf, A.A. (2005) Unsteady Two-Fluid Flow and Heat Transfer in a Horizontal Channel. Heat and Mass Transfer, 42, 81-90. http://dx.doi.org/10.1007/s00231-004-0565-X

[20] Umavathi, J.C., Mateen, A., Chamkha, A.J. and Mudhaf, A.A. (2006) Oscillatory Hartmann Two Fluid Flow and Heat Transfer in a Horizontal Channel. International Journal of Applied Mechanics and Engineering, 11, 155-178.

[21] Vafai, K. and Tien, C.L. (1981) Boundary and Inertia Effects of Flow and Heat Transfer in Porous Media. International Journal of Heat Mass Transfer, 24, 195-203. http://dx.doi.org/10.1016/0017-9310(81)90027-2

[22] Kaviany, M. (1991) Principles of Heat Transfer in Porous Media. Springer-Verlag, New York. http://dx.doi.org/10.1007/978-1-4684-0412-8

[23] Nield, D.A. and Bejan, A. (2006) Convection in Porous Media. Springer-Verlag, New York.

[24] Alkam, M.K., Al-Nimr, M.A. and Hamdan, M.O. (2002) On Forced Convection in Channels Partially Filled with Porous Substrate. Heat and Mass Transfer, 38, 337-342. http://dx.doi.org/10.1007/s002310000177

[25] Rudraiah, N. and Nagraj, S.T. (1977) Natural Convection through Vertical Porous Stratum. International Journal of Engineering Science, 15, 589-600. http://dx.doi.org/10.1016/0020-7225(77)90055-6

[26] Kaviany, M. (1985) Laminar Flow through a Porous Channel, Bounded by Isothermal Parallel Plates. International Journal of Heat Mass Transfer, 28, 851-858. http://dx.doi.org/10.1016/0017-9310(85)90234-0

[27] Backermann, C., Viskanta, R. and Ramadhyani, S. (1988) Natural Convection in Vertical Enclosures Containing Simultaneously Fluid and Porous Layers. The Journal of Fluid Mechanics, 186, 257-284. http://dx.doi.org/10.1017/S0022112088000138

[28] Singh, A.K., Paul, T. and Thorpe T.R. (1999) Natural Convection Due to Heat and Mass Transfer in Composite System. Heat and Mass Transfer, 35, 39-48. http://dx.doi.org/10.1007/s002310050296

[29] Khalili, A., Shivakumara, I.S. and Suma, S.P. (2003) Convective Instability in Superposed Fluid and Porous Layers with Vertical through Flow. Transactions on Porous Media, 51, 1-18. http://dx.doi.org/10.1023/A:1021246000885

[30] Umavathi, J.C., Chamkha, A.J., Mateen, A. and Mudhaf, A.A. (2009) Unsteady Oscillatory Flow and Heat Transfer in a Horizontal Composite Porous Media Channel. Nonlinear Analysis: Modelling and Control, 14, 397-415.

[31] Stamenkovic, M.Z. (2012) Magnetohydrodynamic Flow and Heat Transfer of Two Immiscible Fluids with Induced Magnetic Field Effects. Thermal Science, 16, 33-336. http://dx.doi.org/10.2298/TSCI120430172S

[32] Gupta, U., Jha, A.K. and Chaudhary, R.C. (2011) Free Convection Flow between Vertical Plates Moving in Opposite Direction and Partially Filled with Porous Medium. Applied Mathematics, 2, 935-941. http://dx.doi.org/10.4236/am.2011.28128

[33] Daniel, S. and Daniel, S.Y. (2013) Convective Flow of Two Immiscible Fluid and Heat Transfer with Porous Media along an Inclined Channel with Pressure Gradient. Research Inventy: International Journal of Engineering and Science, 2, 12-18.

[34] Mateen, A. (2013) Magnetohydrodynamic Flow and Heat Transfer of Two Immiscible Fluids through a Horizontal 
Channel. International Journal of Current Engineering and Technology, 3, 1952-1956.

[35] He, J.-H. (2000) A Coupling Method of a Homotopy Technique and a Perturbation Technique for Non Linear Problem. International Journal of Non-Linear Mechanics, 35, 37-43. http://dx.doi.org/10.1016/S0020-7462(98)00085-7

[36] He, J.-H. (2009) An Elementary Introduction to the Homotopy Perturbation Method. Computers and Mathematics with Applications, 57, 410-412. http://dx.doi.org/10.1016/j.camwa.2008.06.003

[37] Biazar, J. and Ghazvini, H. (2008) Homotopy Perturbation Method for Solving Hyperbolic Partial Differential Equations. Computers and Mathematics with Applications, 56, 453-458. http://dx.doi.org/10.1016/j.camwa.2007.10.032

[38] Kim, S. and Russel, W.B. (1985) Modelling of Porous Media by Renormalization of the Stokes Equations. Journal of Fluid Mechanics, 154, 269-286. http://dx.doi.org/10.1017/S0022112085001525

[39] Darcy, H.P.G. (1856) Les Fontaines Publiques de la villa de Dijon. Dalmont, Paris. 